$\begin{array}{cccc}\text { Pendel I } & 248.9668 & \text { Linien des Maassstabs } \\ \text { II } & \mathbf{2 4 8 . 7} \text { 166 } \\ \text { Leichtes Pendel } & \mathbf{2 4 7 . 8 2 5 5} *\end{array}$

Berechnet man dann die Länge des Secundenpendels und reducirt die Linien des Maassstabs auf die Fortin'sche Toise bei $+13^{\circ} \circ \mathrm{R}$., so erhält man:

$$
\begin{aligned}
& \left.\begin{array}{cc}
\text { Pendel I } & 440.1830 \\
\text { II } & 440.1746
\end{array}\right\} \quad 440.1788 \\
& \text { Leichtes Pendel } \quad 440.2116 \\
& \text { Differenz } \frac{40.2116}{0.0328}
\end{aligned}
$$

Nach Berücksichtigung der oben angeführten Gewichte der drei Pendel ergiebt sich dann als Correction für das Mitschwingen des Stativs:

$$
\text { für Pendel I und II }+0.0665 \text { Par. Lin. }
$$$$
\text { für das leichte Pendel }+0.0337 \text {, }
$$

Im Jahre 188I erhielt ich für Pendel I und II durch Schwingen derselben an einer festen Wand (A. N. Bd. ror Nr. 24 I6) die Correction +o.0650 Par. Lin.

Tiflis 1885 Juli.

\title{
Heliographische Oerter von Sonnenflecken im Jahre 1883 beobachtet auf der Sternwarte in Zürich. \\ Von $A$. Wolfer.
}

Im Anschlusse an die in Nr. 2536 der A. N. mitgetheilten. Sonnenfleckenörter für 1882 folgt hier eine entsprechende Uebersicht der im Laufe des Jahres 1883 erhaltenen Resultate für die Rotationsperioden 297 bis 310 incl. Ueber die Art der Beobachtung und Berechnung der Oerter ist das Nöthige in Nr. 53 und 56 der $\gg$ Astron. Mittheilungen « des Herrn Prof. Wolf angegeben; für die Rotationselemente der Sonne habe ich wie bisher die Spörer'schen Werthe angenommen, nämlich

$$
i=7.00 \quad \delta 8=74: 83(\mathrm{r883}) \quad \xi=\mathrm{I} 4^{\circ} .2665 \text {. }
$$

Die Tabellen enthalten die mittleren, auf ganze Grade abgekürzten heliographischen Normallängen $L$ und Breiten $b$

\begin{tabular}{|c|c|c|c|}
\hline Nr. & $L$ & $b$ & Bemerkungen \\
\hline \multicolumn{4}{|c|}{ Rotationsperiode $297 . \quad$ I 882 XII $23-1883$ I 9.} \\
\hline $\mathbf{I}$ & $264^{\circ}$ & $-28^{\circ}$ & Beh. Fleck, I 6 ausgetr. Vgl. R. 298.3 \\
\hline & 259 & -28 & Beh. Fleck, I 6 ausgetr. \\
\hline 2 & $25^{\circ}$ & -25 & Gruppe kl. Flecke, I 6 ausgetr. \\
\hline 3 & 249 & -1 4 & Beh. Fleck, I 7 ausgetr. Vgl. R. 298.5 \\
\hline 4 & 213 & -10 & Beh. Fleck Vor I 8 aufgelöst. \\
\hline & $211-206$ & -10 & Kl. Flecke Vor 18 autgelost. \\
\hline 5 & 192 & -18 & 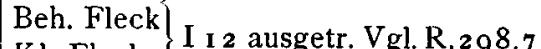 \\
\hline 6 & $\begin{array}{l}183 \\
178\end{array}$ & -20 & Kl. Flecke Kl \\
\hline 0 & $\begin{array}{c}178 \\
172-167\end{array}$ & $\begin{array}{l}+5 \\
+\quad 6\end{array}$ & 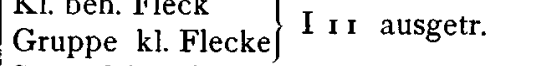 \\
\hline 7 & I 26 & +11 & Sporad. kl. Fleck. \\
\hline 8 & 53 & -8 & Beh. Fleck, I 9 eingetr., I 22 ausgetr. \\
\hline 9 & 26 & +14 & Beh. Fleck, I I 2 eingetr., I 24 ausgetr. \\
\hline 10 & 21 & $-\mathrm{II}$ & Beh. Fleck, ? eingetr., I 24 ausgetr. \\
\hline
\end{tabular}
der Flecken, bei ausgedehnteren Gruppen je die Grenzen des Thätigkeitsbereiches, ferner Bemerkungen über die äussere Erscheinung, sowie die Zeiten der Entstehung und Auflösung, bezw. des Ein- und Austritts der Fleckengruppen. Die detaillirten genauen Orts- und Zeitangaben sind in den Nummern 63 u. f. der "Astron. Mittheilungen * enthalten, auf welche ich hierfür

\begin{tabular}{|c|c|c|c|}
\hline $\mathrm{Nr}$. & $L$ & $b$ & Bemerkungen \\
\hline 6 & $235^{\circ}$ & $+10^{\circ}$ & Beh. Fleck \\
\hline & $235-228$ & $+(\mathbf{I} \mathbf{I} \cdot \mathbf{1} 3)$ & Gruppe kl.Flecke I I 3 I aufg \\
\hline 7 & 187 & -19 & $\begin{array}{l}\text { Beh. Fleck, I } 27 \text { als kl. Fle } \\
\text { If } 4 \text { aufgelöst. Vgl. R. }\end{array}$ \\
\hline 8 & I 32 & +12 & $\begin{array}{l}\text { Grosser beh. Fleck, } \\
\text { I } 3 \text { I eingetr., II I } 2 \text { ausgetr. }\end{array}$ \\
\hline 9 & 81 & -6 & Beh. Fleck / Gruppe II 5 entst., II 9 \\
\hline & 73 & -4 & $\therefore \quad>\quad$ stark entw., II I \\
\hline 10 & $7^{6-6}$ & $+(3-5)$ & $\begin{array}{l}\text { Gruppe mit } 2 \text { beh. Flecken. II } 6 \\
\text { eingetr., II I } 5 \text { aufgelöst. }\end{array}$ \\
\hline I I & 32 & -17 & Beh. Fleck $\left.\right|_{\text {II }}$ \\
\hline & 33 & $-2 \mathrm{I}$ & Kl. Flecke $\}^{11}$ \\
\hline I 2 & 67 & -19 & Sporad. kl. Fleck. \\
\hline I 3 & 19 & +15 & Sporad. kl. Flecke. \\
\hline \multicolumn{4}{|c|}{ Rotationsperiode 299 . II I 6 - III I 5.} \\
\hline $\mathbf{I}$ & $\begin{array}{r}353 \\
350-34\end{array}$ & $\begin{array}{l}+8 \\
+8\end{array}$ & $\left.\begin{array}{l}\text { Grosse beh. Kerngr. } \\
\text { Veränd. kl. Flecke }\end{array}\right\} \begin{array}{l}\text { II I eingetr., } \\
\text { II } 3 \text { ausgetr. }\end{array}$ \\
\hline 2 & $330-317$ & -15 & $\begin{array}{l}\text { Gruppe kl. Flecke, } \\
\text { II I } 3 \text { eingetr., II } 23 \text { aufgelöst. }\end{array}$ \\
\hline 3 & 358 & -20 & Sporad. kl. Fleck. \\
\hline 4 & $285-282$ & - 19 & Kl. Flecke. II 18 entst., II 2 I aufgel. \\
\hline 5 & 258 & $-\mathbf{I} 7$ & Sporad. kl. Fleck. \\
\hline 6 & 234 & $-\mathbf{I} \mathbf{I}$ & kl. Fleck. \\
\hline 7 & 233 & -29 & l. kl. Fleck. \\
\hline 8 & 221 & -12 & Beh. Fleck m. 2 Kernen] II 24 \\
\hline & $220-21$ & $-(10.14)$ & $\begin{array}{l}\text { Kl. Flecke } \\
\text { III } 5 \text { als beh. Fleck ausgetr. }\end{array}$ \\
\hline
\end{tabular}
verweise.

Rotationsperiode 298 . I 19 . II 16.

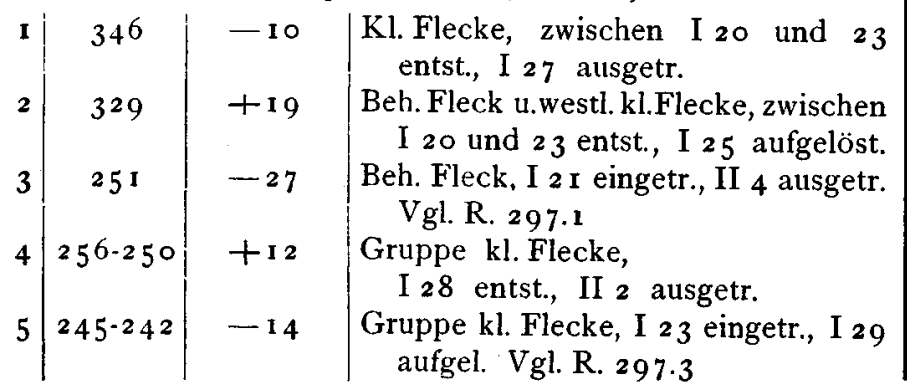




\begin{tabular}{|c|c|c|c|}
\hline $\mathrm{Nr}$. & $L$ & $b$ & Bemerkungen \\
\hline 9 & $172^{\circ}$ & $+19^{\circ}$ & Sporad. kl. Fleck. \\
\hline 10 & $165-161$ & +5 & $\begin{array}{l}\text { Gruppe kl. und beh. Flecke, } \\
\text { II } 25 \text { eingetr., III } 3 \text { aufgelöst. }\end{array}$ \\
\hline I I & 75 & -17 & $\begin{array}{l}\text { Gruppe kl. Flecke, } \\
\text { III } 8 \text { entst., III I } 5 \text { ausgetr. }\end{array}$ \\
\hline 12 & 59 & +13 & Sporad. kl. Fleck. \\
\hline I 3 & 34 & -13 & Beh.Fleck, III 6 eingetr., III I 4 aufgel. \\
\hline 14 & 24.20 & +15 & $\begin{array}{l}\text { Gruppe kl. Flecke, III } 8 \text { als kl. beh. } \\
\text { Fleck eingetr., III I } 3 \text { stark entw., } \\
\text { III I } 7 \text { aufgel. } \\
\text { Sporad. kl. Fleck. }\end{array}$ \\
\hline \multicolumn{4}{|c|}{ Rotationsperiode 300 . III I5-IV 11.} \\
\hline $\mathbf{I}$ & $35^{1}$ & -13 & $\begin{array}{l}\text { Beh. Fleck, zwischen III } 9 \text { und } 13 \\
\text { eingetr., III } 22 \text { ausgetr. }\end{array}$ \\
\hline 2 & 329 & +13 & $\begin{array}{l}\text { Als beh. Fleck III i } 2 \text { eingetr., nach } \\
\text { III } 20 \text { aufgel. oder ausgetr. }\end{array}$ \\
\hline 3 & 307 & +17 & Sporad. kl. Flecke. \\
\hline 4 & $\begin{array}{c}269 \\
263 \cdot 260\end{array}$ & $\begin{array}{l}-8 \\
-(7-9)\end{array}$ & 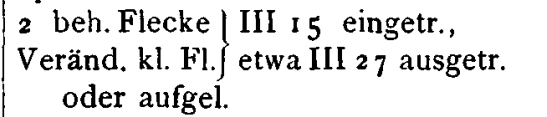 \\
\hline 5 & $267-258$ & -18 & $\begin{array}{l}\text { Gruppe kl. Flecke mit Hofbildungen, } \\
\text { zwischen III I } 7 \text { und I } 9 \text { eingetr. } \\
\text { oder entst., vor III } 28 \text { ausgetr. } \\
\text { oder aufgel. }\end{array}$ \\
\hline 6 & 242 & -19 & $\begin{array}{l}\text { Kl. Fleck, vor III } 28 \text { entst., III } 30 \\
\text { ausgetr. oder aufgel. }\end{array}$ \\
\hline 7 & 225 & -23 & Beh. Fleck J etwa III 2 I \\
\hline & $222-217$ & -24 & $\begin{array}{l}\text { Kleine u. kl. beh. Flecke\} als grosse } \\
\text { Gruppe eingetr., III } 31 \text { ausgetr. } \\
\text { Vgl. R. 30 I. Io }\end{array}$ \\
\hline 8 & 192 & -12 & Sporad. Gruppe von Poren. \\
\hline 9 & 168 & +4 & Sporad. kl. Fleck. \\
\hline 10 & $151-147$ & +7 & $\begin{array}{l}\text { Gruppe von Poren, } \\
\text { III } 3 \text { I entst., IV } 4 \text { aufgel. }\end{array}$ \\
\hline 1 I & 143 & -8 & $\begin{array}{l}\text { Beh. Fleck, } \\
\quad \text { vor III } 28 \text { eingetr., IV } 6 \text { ausgetr. }\end{array}$ \\
\hline 12 & $131-125$ & +8 & Kl.Fleck, IV 5 entst., IV 8 ausgetr. \\
\hline 13 & 107 & +14 & $\begin{array}{l}\text { Gross. beh. Fleck, } \\
\text { III } 28 \text { eingetr., IV } 9 \text { ausgetr. }\end{array}$ \\
\hline 14 & $104-92$ & $+(11-15)$ & $\begin{array}{l}\text { Stark entwick. Gruppe kl. Flecke, } \\
\text { III } 29 \text { eingetr., IV } 9 \text { ausgetr. }\end{array}$ \\
\hline 15 & 95 & -13 & $\begin{array}{l}\text { Beh. Fleck, } \\
\text { III } 29 \text { eingetr., IV } 9 \text { ausgetr. }\end{array}$ \\
\hline 16 & 86 & +8 & Kl. Fleck, III 30 eingetr., IV I aufgel. \\
\hline 17 & 85 & -7 & d. kl. Fleck. \\
\hline 1.8 & $\begin{array}{c}74 \\
74^{-66}\end{array}$ & $\begin{array}{l}+3 \\
+3\end{array}$ & $\begin{array}{l}\text { Beh. Fleck } \\
\text { Gruppe kl. Flecke\} }\} \text { III } 3 \text { entwick., IV } 7 \text { ab- } \\
\text { nehmend, V I I als kl.Fleck ausgetr. } \\
\text { oder aufgel. Vgl. R. 30 I. I }\end{array}$ \\
\hline 19 & $\begin{array}{c}64 \\
64-56 \\
55\end{array}$ & $\begin{array}{r}-17 \\
-18 \\
-17\end{array}$ & \begin{tabular}{l|l}
$\begin{array}{l}\text { Beh. Fleck } \\
\text { Veränd. kl. Fl. }\end{array}$ & IV I eingetr., IV I 2 \\
ausgetr., der östl. beh. \\
$\begin{array}{c}\text { Beh. Fleck } \\
\text { IV } 7 \text { in kl.Flecke zerf. Vgl.R.3० I.24 }\end{array}$ \\
Fleck IV 3 entstand.,
\end{tabular} \\
\hline 20 & 36 & -8 & Sporad. kl. Fleck. \\
\hline 2 I & 6 & -13 & $\begin{array}{l}\text { Gruppe m. beh. Fleck, IV } 7 \text { entst., } \\
\text { IV I } 6 \text { als } \mathrm{kl} \text {. Fleck ausgetr. }\end{array}$ \\
\hline
\end{tabular}

\begin{tabular}{|c|c|c|c|}
\hline Nr. & $L$ & $b$ & Bemerkungen \\
\hline \multicolumn{4}{|c|}{ Rotationsperiode $301 . \quad$ IV II-V 8.} \\
\hline $\mathbf{I}$ & $326^{\circ} \cdot 3$ & $+\left(7^{\circ}\right.$ & $\begin{array}{l}\text { Gruppe m. } 5 \text { beh. u. zahlr. kl. Flecken, } \\
\text { wahrsch. IV } 8 \text { eingetr. oder entst., } \\
\text { IV } 20 \text { ausgetr. }\end{array}$ \\
\hline 2 & 278 & -22 & Grosser beh. Fleck IV I I als Gr. \\
\hline & $277-268$ & $-(20 \cdot 24)$ & $\begin{array}{l}\text { Veränd. kl. u. beh. Fl. kl. Fl. eingetr. } \\
\text { IV } 24 \text { ausgetr. Vgl. R. 302. IO }\end{array}$ \\
\hline \multirow[t]{2}{*}{3} & 274 & -15 & Gross. beh. Fleck IV I I eingetr., \\
\hline & 272 & -16 & Veränd. Gruppe ] IV 24 ausgetr. \\
\hline 4 & 270 & -4 & Sporad. kl. Fleck. \\
\hline 5 & $271-266$ & $+(7 \cdot 9)$ & $\begin{array}{l}\text { Gruppe kl. Flecke, IV I } 6 \text { entst., I } 9 \\
\text { aufgel., } 2 \text { I Neubildung, } 22 \text { ausgetr. }\end{array}$ \\
\hline 6 & 265 & -22 & Sporad. kl. Gruppe. \\
\hline \multirow[t]{2}{*}{7} & 254 & -15 & Norm. beh. Fleck $\quad I_{1} V_{3}$ eingtr. \\
\hline & $25^{2-244}$ & $-(16-18)$ & Veränd. k]. u.beh.Flecke\}IV 2 \\
\hline 8 & 258 & -23 & kl. F \\
\hline 9 & $239-231$ & $-(4-6)$ & $\begin{array}{l}\text { Gruppe, westl. beh. Fleck m. a Kernen, } \\
\text { IV } 17 \text { entst., IV } 26 \text { als norm. beh. } \\
\text { Fleck ausgetr. }\end{array}$ \\
\hline 10 & 224 & -24 & $\begin{array}{l}\text { Kl. Fleck, IV I } 5 \text { beh., IV } 15 \text { eingetr., } \\
\text { IV } 20 \text { aufgel. Vgl. R. } 300.7\end{array}$ \\
\hline I I & 183 & +5 & $\begin{array}{l}\text { Beh. Fleck, IV } 22 \text { entst., } 29 \text { aufgel. } \\
\text { oder als kl. Fleck ausgetr. }\end{array}$ \\
\hline 12 & $178-170$ & $-(15-20)$ & $\begin{array}{l}\text { Gruppe kl. und theilweise beh.Flecke, } \\
\text { IV } 22 \text { entst., } 29 \text { ausgetr. }\end{array}$ \\
\hline 13 & 159 & +4 & IV 2 I eingetr., dann aufgel. \\
\hline 14 & $15 I-146$ & -16 & Kl.Fleck, IV 2 I eingetr. u. rasch aufgel. \\
\hline I 5 & 142 & +8 & $\begin{array}{l}\text { Beh. Fleck, } \\
\text { IV } 22 \text { eingetr., } V_{3} \text { ausgetr. }\end{array}$ \\
\hline 16 & $126 \cdot 133$ & +6 & $\begin{array}{l}\text { Gruppe kl. Flecke, } \\
\text { IV } 23 \text { eingetr., IV } 29 \text { aufgel. }\end{array}$ \\
\hline 17 & -118 & + & $\begin{array}{l}\text { Gruppe kl. Flecke, } \\
\mathrm{V}_{3} \text { entst., } \mathrm{V}_{5} \text { ausgetr. }\end{array}$ \\
\hline I 8 & I I I & +13 & $\begin{array}{l}\text { Kl. beh. Fleck, } \\
\text { IV } 24 \text { eingetr., } V_{2} \text { aufgel. }\end{array}$ \\
\hline 19 & 0 & -14 & Sporad. kl. Fleck. \\
\hline 20 & 86 & -19 & $\mathrm{Sp}$ \\
\hline 21 & $80-75$ & +6 & $\begin{array}{l}\text { Kl. Flecke, wahrscheinl. IV } 30 \text { entst., } \\
\text { V } 6 \text { aufgel. Vgl. R. } 300.18\end{array}$ \\
\hline 22 & 79 & +13 & K1. Fleck, V 7 entst., 8 ausgetr. \\
\hline 23 & 74 & + & $\begin{array}{l}\text { Gruppe kl. Flecke, V } 28 \text { eingetr., dann } \\
\text { aufgel., V } 2 \text { Neubildung. }\end{array}$ \\
\hline 24 & 68 & - I 5 & $\begin{array}{l}\text { Beh. Fleck, IV } 27 \text { eingetr., } 29 \text { aufgel., } \\
\text { V } 4 \text { Neubildung, V } 5 \text { aufgel. } \\
\text { Vgl. R. 300.19 }\end{array}$ \\
\hline 25 & 5 & 12 & Sporad. kl. Fleck. \\
\hline 20 & & $+\left(I_{4}-1\right.$ & $\begin{array}{l}\text { Gruppe mit beh. Fleck westlich, } \\
V_{7} \text { entst., } V_{\text {I } 3} \text { ausgetr. od. aufgel. }\end{array}$ \\
\hline
\end{tabular}

Rotationsperiode $302 . \quad \mathrm{V} 8-\mathrm{VI}_{4}$.

\begin{tabular}{c|c|c|c|} 
I & 359 & -16 & Sporad. kl. Fleck. \\
$\mathbf{2}$ & $357-353$ & $+(\mathbf{I}-12)$ & Gruppe m. beh. Fleck westl., V6 entst.,
\end{tabular} zwischen V I I U. I5 aufgel, oder ausgetr.

$337-18$
Beh. Fleck, $\mathrm{V}_{4}$ eingetr., $\mathrm{V}_{\mathrm{I}} 6$ als $\mathrm{kl}$. Fleck ausgetr. 


\begin{tabular}{|c|c|c|c|}
\hline $\mathrm{Nr}$. & $L$ & $b$ & Bemerkungen \\
\hline 4 & $331^{\circ}$ & $+12^{\circ}$ & Sporad. Gruppe kl. Flecke. \\
\hline 5 & $327-324$ & $-(15-17)$ & $\begin{array}{l}\text { Gruppe kl. Flecke, } \\
V_{5} \text { eingetr., } V_{7} \text { aufgel. }\end{array}$ \\
\hline 6 & 289.285 & $-\mathrm{I} 5$ & $\begin{array}{l}\text { Gruppe mit beh. Fl. westlich, } \\
\text { zwischen V I I und I } 5 \text { entst., } \\
V_{2} \text { o ausgetr. Vgl. R. } 303.5\end{array}$ \\
\hline 7 & 286 & -6 & $\begin{array}{l}\text { Gruppe kl. Flecke, zwischen V I I } \\
\text { und I } 5 \text { entst., I } 7 \text { aufgel. }\end{array}$ \\
\hline 8 & 273 & -13 & Sporad. kl. Fleck. \\
\hline 9 & 271 & -15 & $\begin{array}{l}\text { Kl. Fleck, } \\
\text { V } 9 \text { eingetr., nach } V_{\text {I I }} \text { aufgel. }\end{array}$ \\
\hline 10 & 268 & -22 & $\begin{array}{l}\text { Beh. Fleck, östl. kl. Fl., V } 9 \text { eingetr., } \\
V_{2} \text { I ausgetr. Vgl. } 30 \text { I.2 u. } 303.6\end{array}$ \\
\hline II & 260 & +11 & $\begin{array}{l}\text { Beh. Fleck, östl kleine Flecke, } \\
\text { V } 9 \text { eingetr., V } 22 \text { ausgetr. }\end{array}$ \\
\hline 12 & 248 & -22 & $\begin{array}{l}\text { Gruppe kl. Flecke, } \\
\text { V i } 8 \text { entst., } V_{22} \text { aufgel. }\end{array}$ \\
\hline I 3 & 190 & -6 & $\begin{array}{l}\text { Gruppe kl. Flecke, } \\
V_{23} \text { entst., V } 25 \text { aufgel. }\end{array}$ \\
\hline 14 & $195^{-187}$ & +21 & $\begin{array}{l}\text { Gruppe kl. Flecke, V I } 5 \text { eingetr. } \\
\text { oder entst., V } 22 \text { aufgel. }\end{array}$ \\
\hline 15 & 180 & +10 & $\begin{array}{l}\text { Gruppe kl. Flecke, } \\
\qquad 2_{3} \text { entst., V } 25 \text { aufgel. }\end{array}$ \\
\hline 16 & $84-8 r$ & $+(13-15)$ & $\begin{array}{l}\text { Gruppe kl. Flecke, } \\
\text { V } 24 \text { eingetr., } V_{27} \text { aufgel. }\end{array}$ \\
\hline 17 & $67-62$ & $+(14 \cdot 17)$ & $\begin{array}{l}\text { Gruppe kl. Flecke, } \\
\text { VI } 4 \text { entst., VI } 6 \text { ausgetr. }\end{array}$ \\
\hline 18 & $\begin{array}{c}40 \\
40-34\end{array}$ & $\begin{array}{c}-7 \\
-(5-9)\end{array}$ & $\left.\begin{array}{l}\text { Gross. beh. Fleck } \\
\text { Veränd. kl. u. beh. Fl. }\end{array}\right\}$ VI I 8 ausgetr. \\
\hline & 32 & - I0 & Gr. beh. Kerngruppe Jvgl. R.303.20 \\
\hline 19 & $3^{6-33}$ & +8 & $\begin{array}{l}\text { Gruppe kl. Flecke, } \\
\text { VI } 2 \text { entst., VI } 3 \text { aufgel. }\end{array}$ \\
\hline 20 & 30 & +17 & Kl. Fleck, VI 7 entst., VI 8 ausgetr. \\
\hline \multicolumn{4}{|c|}{ Rotationsperiode $3 \circ 3$. VI 4 -VII $\mathrm{r}_{\text {. }}$} \\
\hline $\mathbf{I}$ & $\begin{array}{c}343 \\
343-340 \\
339\end{array}$ & $\begin{array}{l}+14 \\
+(12.15) \\
+15\end{array}$ & $\left.\begin{array}{l}\text { Beh. Fleck } \\
\text { Kl. Flecke } \\
\text { Beh. Fleck }\end{array}\right\}$ VI 3 i 0 eingetr., \\
\hline 2 & $34^{6-33} \mathrm{I}$ & $-(10-17)$ & $\begin{array}{l}\text { Gruppe kl. Flecke, } \\
\text { VI }_{4} \text { entst., VI I } 2 \text { ausgetr. }\end{array}$ \\
\hline 3 & $32 I \cdot 316$ & +7 & $\begin{array}{l}\text { Gruppe mit kl. beh. Fl. westl., } \\
\text { VI } 6 \text { entst., vor VI I I aufgel. }\end{array}$ \\
\hline 4 & $321 \cdot 3 \circ 7$ & $-(7-12)$ & Stark entw. Gruppe $\quad$ VI 2 als beh. \\
\hline & 313 & -10 & $\begin{array}{l}\text { Beh.Fleck m. } 2 \text { Kernen\} Fleck eingtr., } \\
\text { nach VI I I aufgel. }\end{array}$ \\
\hline 5 & 293 & -16 & $\begin{array}{l}\text { Beh. Fleck, VI } 3 \text { eingetr., zwischen } \\
\text { VI } 8 \text { u. I I aufgel. Vgl. R. } 302.6\end{array}$ \\
\hline 6 & $261 \cdot 25^{8}$ & $-(22 \cdot 26)$ & $\begin{array}{l}\text { Beh. Kerngruppe u. kl. Flecke, } \\
\text { VI } 6 \text { eingetr., VI I } 8 \text { ausgetr. } \\
\text { Vgl. R. 302.10 und R. 304.2 }\end{array}$ \\
\hline 7 & 223 & -16 & Kl.Fleck, VI 18 entst., VI 20 ausgtr. \\
\hline 8 & $206-20$ & -25 & $\begin{array}{l}\text { Gruppe mit kl. beh. Fl. westl., } \\
\text { VI } 1 \text { o eingetr., nach VI I } 5 \text { aufgel. }\end{array}$ \\
\hline 9 & $185-172$ & $-(22-27)$ & $\begin{array}{l}\text { Stark entw. Gruppe m. } 3 \text { beh. Fleck., } \\
\text { zw.VI I } 5 \text { u. } 18 \text { entst., VI } 24 \text { ausgtr. } \\
\text { Vgl. R. 304. } 5\end{array}$ \\
\hline 10 & I 66 & +21 & Beh.Fleck, VI I 4 eingtr.,VI I 9 aufgel: \\
\hline
\end{tabular}

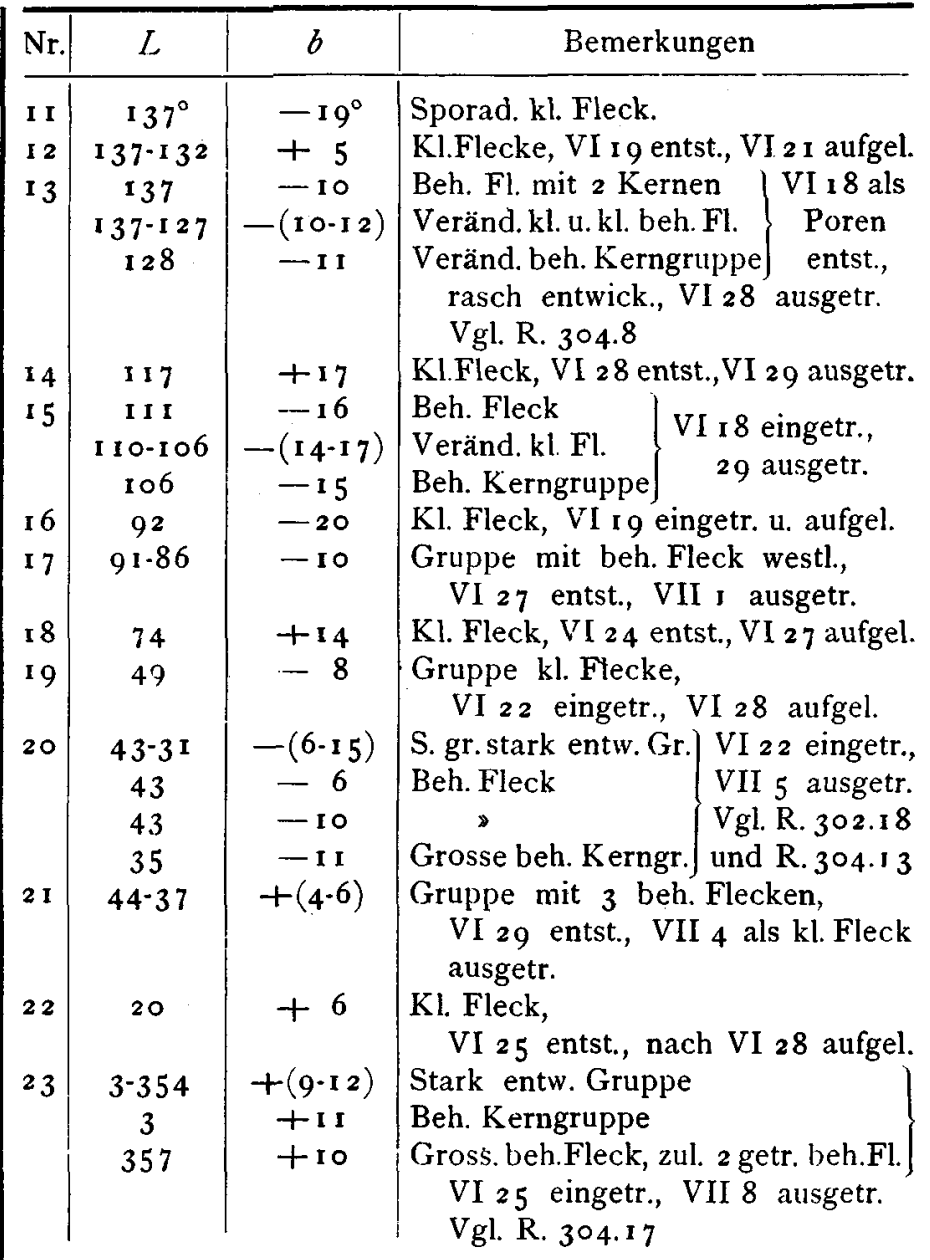

Rotationsperiode 304. VII I - VII 28.

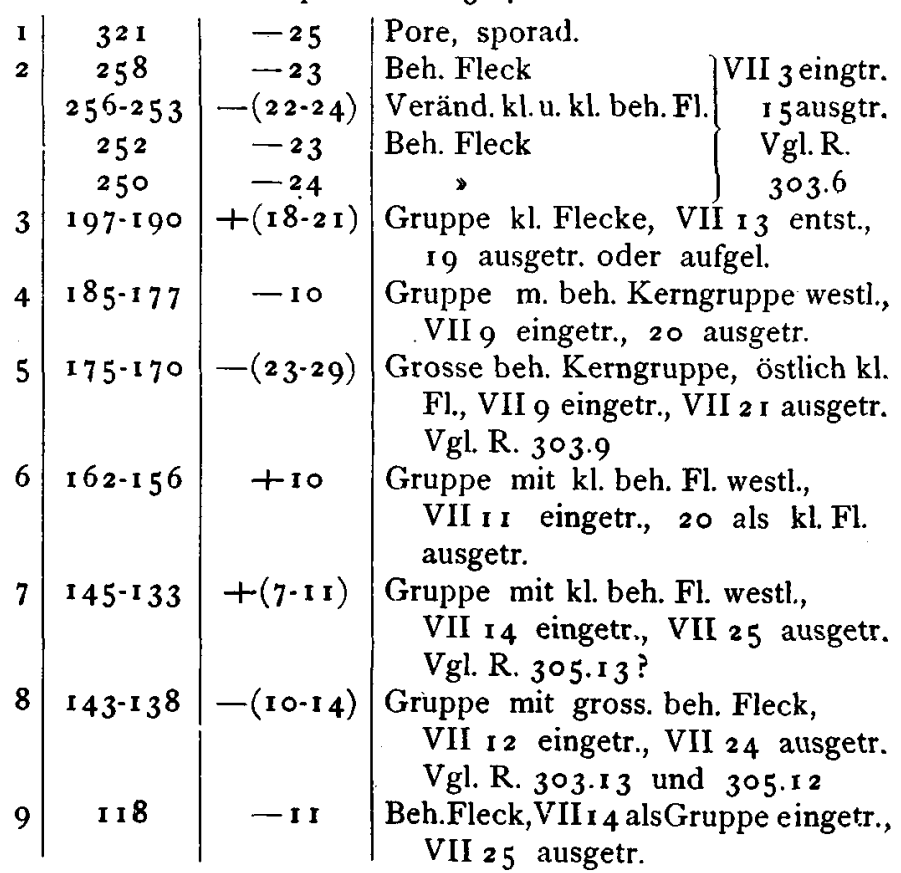




\begin{tabular}{|c|c|c|c|}
\hline $\mathrm{Nr}$. & $L$ & $b$ & Bemerkungen \\
\hline 10 & $99^{\circ}-88^{\circ}$ & $-\left(14^{\circ} \cdot 17^{\circ}\right.$ & $\begin{array}{l}\text { Gruppe mit } 2 \text { beh. } \\
\text { VII I } 6 \text { eingetr., }\end{array}$ \\
\hline 11 & $\begin{array}{c}74 \\
73^{-66}\end{array}$ & $\begin{array}{l}-16 \\
-16\end{array}$ & \begin{tabular}{l|l} 
Beh. Fleck & VII i 7 ei \\
Kl. beh. u. kl. Fl. & VII 28 au
\end{tabular} \\
\hline 12 & $62-5$ & +5 & $\begin{array}{l}\text { Gruppe kl. Flecke, } \\
\text { VII } 19 \text { entst., } 26 \text { aufgel. }\end{array}$ \\
\hline 13 & $\begin{array}{c}47-35 \\
34\end{array}$ & $\begin{array}{l}-(5-13) \\
-10\end{array}$ & $\begin{array}{l}\text { Sehr stark entw. Gruppe } \\
\text { Grosses Hofgebilde mit } 3 \text { Kernf.) } \\
\text { VII I9 eingetr., VIII I ausgetr. } \\
\text { Vgl. R. } 303.20 \text { und } 305.23 \text { (?) }\end{array}$ \\
\hline 14 & 3 & +9 & $\begin{array}{l}\text { Beh. Fleck, östl. kl. Fl. } \\
\text { VII i } 9 \text { eingetr., VII } 27 \text { aufgel. }\end{array}$ \\
\hline 15 & $3^{I-2}$ & $5-18)$ & $\begin{array}{l}\text { Gruppe kl. Flecke, } \\
\text { VII } 24 \text { entst., nach VII } 27 \text { aufgel. }\end{array}$ \\
\hline & 8 & & \\
\hline 1 & 4 & & $\begin{array}{l}\text { Norm. beh. Fleck, VII } 22 \text { eingetr., } \\
\text { VIII } 3 \text { ausgetr. Vgl. R. } 3^{\circ} 3.23\end{array}$ \\
\hline
\end{tabular}

Rotationsperiode 305. VII 28 - VIII 24.

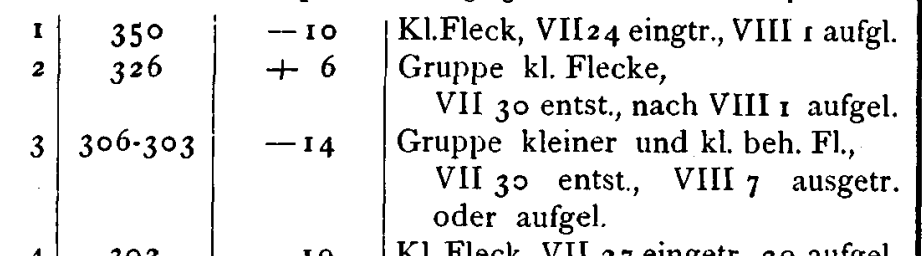

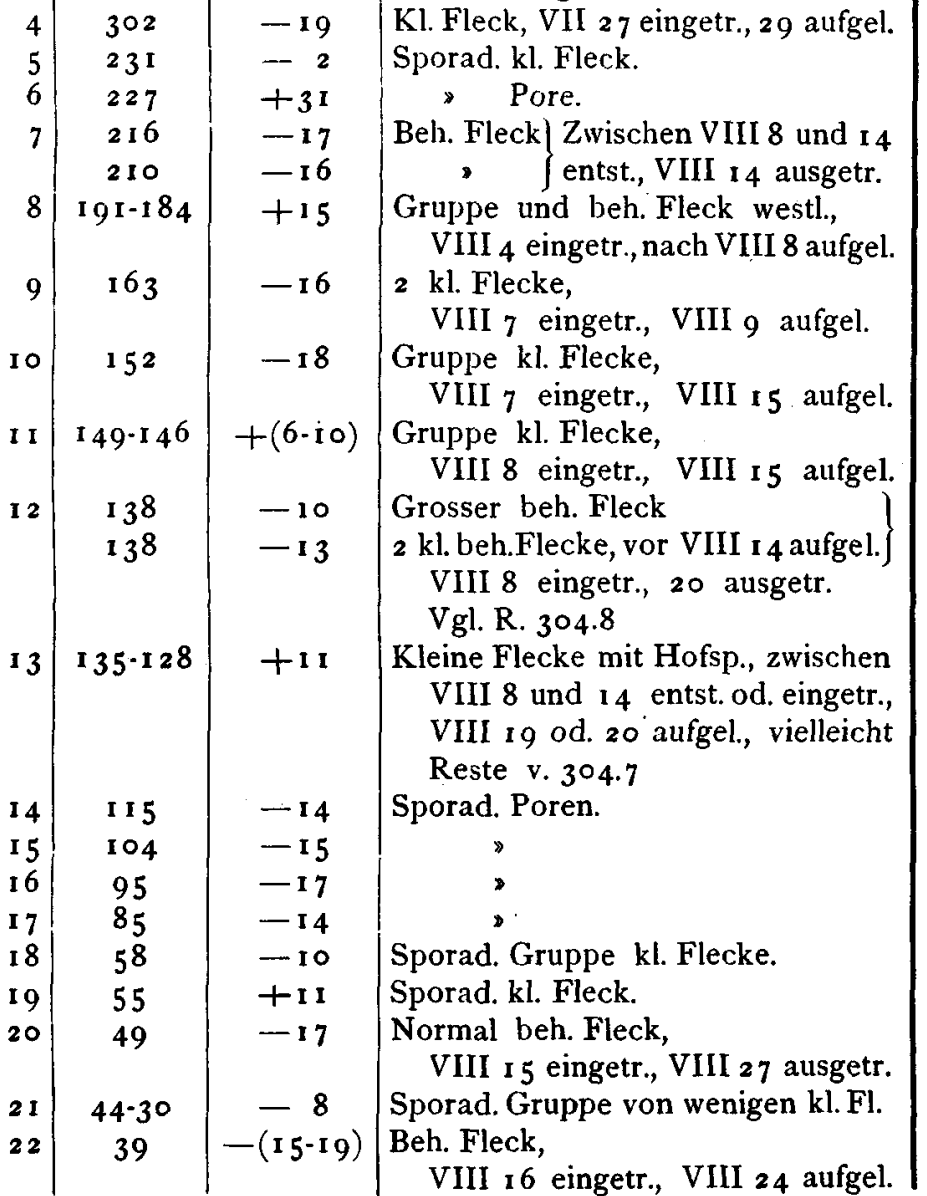

\begin{tabular}{|c|c|c|c|}
\hline $\mathrm{Nr}$. & $L$ & $b$ & Bemerkungen \\
\hline 23 & $34^{\circ}-30^{\circ}$ & $-\left(8^{\circ}-13^{\circ}\right)$ & $\begin{array}{l}\text { Gruppe kl. Flecke, VIII I } 6 \text { eingetr., } \\
25 \text { aufgel., vielleicht Reste von } \\
\text { R. } 304.13 \text {. }\end{array}$ \\
\hline 24 & 29 & $\circ$ & $\begin{array}{l}\text { Gruppe kl. Flecke, } \\
\text { VIII i } 6 \text { eingetr., VIII } 20 \text { aufgel. }\end{array}$ \\
\hline 25 & 24 & +10 & $\begin{array}{l}\text { Gruppe kl. Flecke, } \\
\text { VIII i } 9 \text { entst., } 22 \text { aufgel. }\end{array}$ \\
\hline 26 & 23.20 & -17 & $\begin{array}{l}\text { KI. Fleck, } \\
\text { VIII } 24 \text { entst., VIII } 26 \text { aufgel. }\end{array}$ \\
\hline 27 & $18-10$ & $+(9-12)$ & $\begin{array}{l}\text { VIII } 17 \text { eingetr. Gruppe kl. Flecke, } \\
22 \text { aufgel., } 25 \text { Neubild., } 28 \text { aufgel., }\end{array}$ \\
\hline 28 & 12 & -9 & $\begin{array}{l}\text { Gruppe kl. Flecke, } \\
\text { VIII } 22 \text { entst., } 25 \text { aufgel. }\end{array}$ \\
\hline
\end{tabular}

Rotationsperiode 306 . VIII 24 - IX 20.

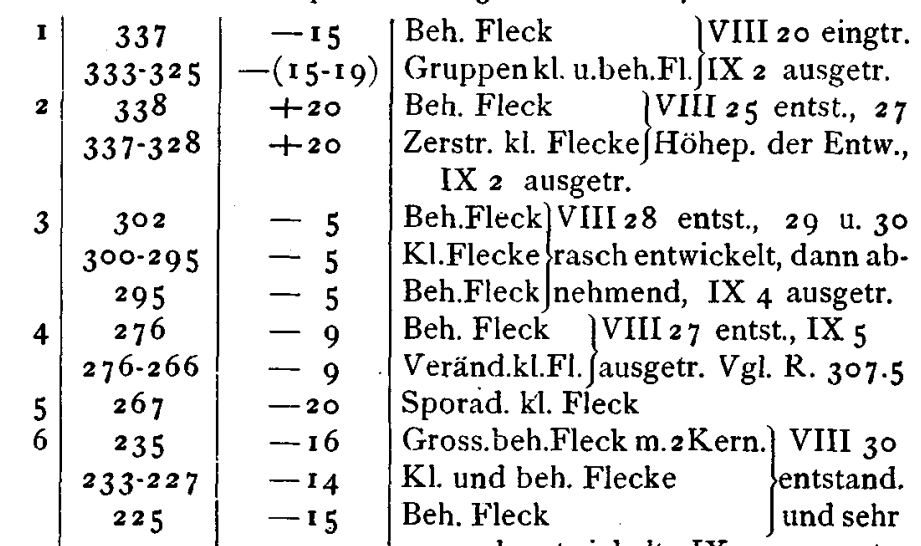
rasch entwickelt, IX IO ausgetr.
\begin{tabular}{l|l|l|l}
7 & $\mathbf{2} 20$ & -15 & Sporad. kl. Flecke. \\
8 & 2 1 2 & -19 & Grosser beh. Fleck mit 3 Kernen,
\end{tabular} zuletzt 2 getrennte Hofflecke, VIII 29 eingetr., IX II ausgetr. Vgl. R. 307.9
Gruppe kl. Flecke, IX I eingetr., IX I 3 ausgetr.
Beh. Fleck, später 2 getr. Hofflecke)
$\mathrm{Kl}$. beh. und $\mathrm{kl}$. Flecke IX I eingetr., 14 ausgetr.

Sporad. Pore

Kl. beh. und kl. Fleck, IX 2 eingetr., IX 6 aufgel. Sporad. kl. Fleck.

Kl. Fleck, IX 3 eingetr., IX 5 aufgel. Gruppe kl. Flecke,

IX 12 entst., IX 15 ausgetr.

Gross.beh.Fleck m.2 Kernen IX 7

Gruppen kl Flecke Geingtr., IX 20 ausgetr. Vgl. R. 307.19

Beh. Fleck I IX 13 entst.,

Gruppen kl. Flecke\} IX 19 ausgetr.

Gruppen kl. Flecke, IX I I entst., IX 15 aufgel.

Beh. Fleck IX 10 eingetr., Gruppe kl. Flecke\} IX I 9 aufgel. Sporad. kl. Flecke. 


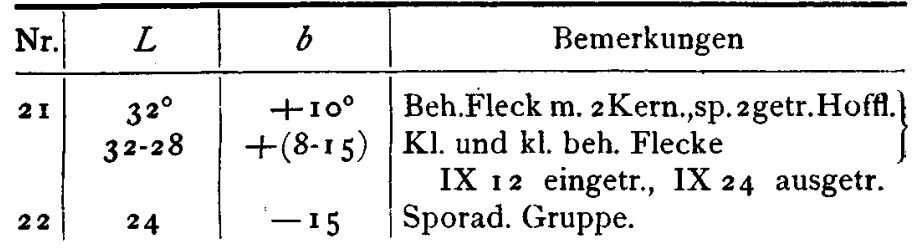

Rotationsperiode $307 . \quad$ IX $20-X \quad 18$.

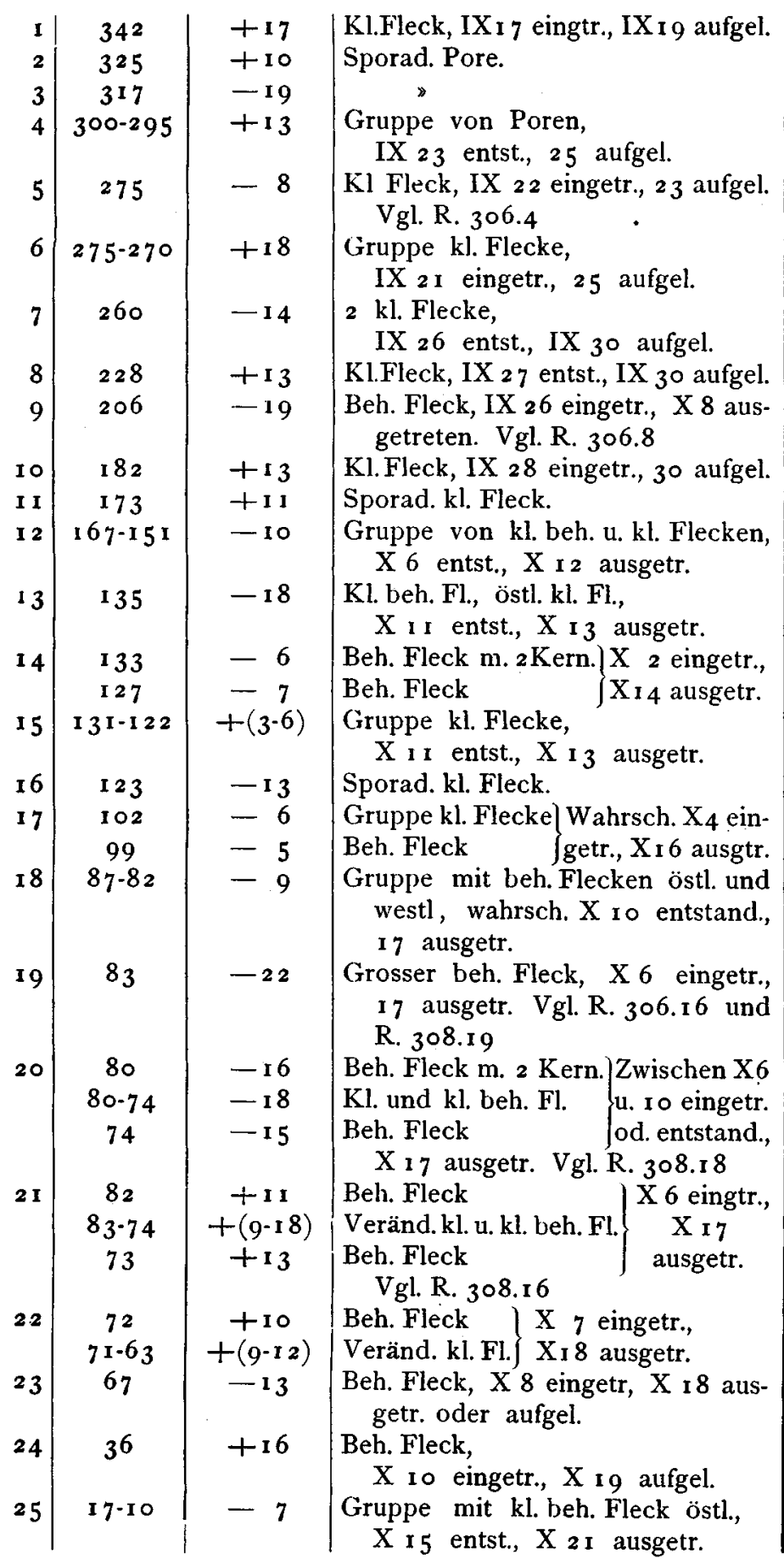

\begin{tabular}{|c|c|c|c|}
\hline Nr. & $L$ & $b$ & Bemerkungen \\
\hline \multicolumn{4}{|c|}{ Rotationsperiode $308 . \quad X_{18-X I} 4}$. \\
\hline $\mathbf{I}$ & $\begin{array}{c}35^{\circ} \\
349-345 \\
344\end{array}$ & $\begin{array}{l}-10^{\circ} \\
-(6-11) \\
-8\end{array}$ & $\begin{array}{l}\text { Sehr gross. beh. Fleck } \\
\text { Veränd. kl. und beh. Fl. } \\
\text { Beh. Fleck } \\
\quad \text { Vgl. R. 309.2-3 }\end{array}$ \\
\hline 2 & 343.337 & $+(18-21)$ & $\begin{array}{l}\text { Veränd. kl. Flecke, X I } 8 \text { entstand., } \\
20 \text { aufgel., } 2 \text { I od. } 22 \text { Neubildung. }\end{array}$ \\
\hline 3 & 335 & +12 & \multirow{4}{*}{$\begin{array}{l}\text { Kl. theilweise beh. Fleck, } \\
\text { X } 13 \text { eingetr., X I } 9 \text { aufgel. } \\
\text { Gruppe kl. Flecke, } \\
\text { wahrsch. X } 23 \text { entst., } 27 \text { ausgetr. } \\
\text { Gruppe kl. u. beh.Flecke, X20 entst., } \\
29 \text { ausgetr. Vgl. R. } 309.5 \\
\text { Sporad. kl. Fleck. }\end{array}$} \\
\hline 4 & 310 & +14 & \\
\hline 5 & $283-273$ & $-\left(9^{-15}\right)$ & \\
\hline 6 & 266 & -7 & \\
\hline 7 & $23^{6}$ & +15 & \multirow{2}{*}{$\begin{array}{l}\text { Beh. Fleck, Östl. kl. Fl., } \\
\text { wahrsch. X } 2 \text { I eingtr.,XI I ausgtr. } \\
\text { Beh. Fleck, X } 23 \text { eingetr., XI } 4 \text { als } \\
\text { kl. Fl. ausgetr. }\end{array}$} \\
\hline 8 & 208 & -7 & \\
\hline 9 & $192-172$ & $-(7-12)$ & \multirow{3}{*}{$\begin{array}{l}\text { Sehr ausged. Gruppe } \mid \mathrm{X}_{2} 5 \text { eingetr., } \\
\text { Beh. Fleck m. } 2 \text { Kern. XI } 6 \text { ausgetr. } \\
\text { Vgl. R. } 3 \circ 9 .{ }^{\circ} 2 \\
\text { Gruppe mit beh. Fleck westlich, } \\
\text { zwischen X } 28 \text { und XI I entst., } \\
\text { XI } 6 \text { ausgetr. }\end{array}$} \\
\hline & 177 & -9 & \\
\hline 0 & $185-180$ & +22 & \\
\hline $\mathbf{I}$ & $164-152$ & $-\mathbf{r} \cdot \mathbf{1}$ & $\begin{array}{l}\text { Gruppe kl. und kl. beh. Flecke, vor } \\
\text { XI I entstanden, XI } 7 \text { ausgetr. } \\
\text { Vgl. R. 3०9.1 } 4\end{array}$ \\
\hline 2 & 148 & +14 & $\begin{array}{l}\text { Beh. Fleck mit } 2 \text { Kernen, } \\
\mathrm{X}_{2} 8 \text { eingetr., XI } 2 \text { aufgel. }\end{array}$ \\
\hline 13 & I $40-13^{\circ}$ & $+(2 \cdot 5)$ & \multirow{3}{*}{$\begin{array}{l}\text { Gruppe mit beh. Fleck westl., } \\
\text { X } 28 \text { eingetr., XI Io ausgetr. } \\
\text { Sporad. Pore. } \\
\text { Kl. Fleck, } \\
\text { vor XI i eingetr.,nach XI ` aufgel. }\end{array}$} \\
\hline 4 & I 28 & +15 & \\
\hline 15 & II & +1 & \\
\hline 6 & $88-79$ & $+\left(9^{-1} 5\right)$ & $\begin{array}{l}\text { Gruppe m.4 beh.Flecken,XI I eingtr., } \\
\text { I } 2 \text { aufgel. Vgl. R. } 307.2 \text { I }\end{array}$ \\
\hline 7 & 88.83 & -11 & \multirow{2}{*}{$\begin{array}{l}\text { Gruppe kl. Flecke, } \\
\text { XI I eingetr., nach XI } 5 \text { aufgel. } \\
\text { Beh. Fleck, XI I eingetr., nach XI } 5 \\
\text { aufgel. Vgl. R. } 3 \circ 7.20\end{array}$} \\
\hline 18 & 84 & -17 & \\
\hline 19 & 74 & -23 & $\begin{array}{l}\text { Beh.Fleck, XI I eingetr., XI I } 4 \text { aus- } \\
\text { getr. Vgl. R. 307.1 } 9\end{array}$ \\
\hline 20 & 25.19 & $+(9-13)$ & \multirow{2}{*}{$\begin{array}{l}\text { Gruppe kl. Flecke, } \\
\text { XI I o entst., XI I } 6 \text { aufgel. } \\
\text { Gruppe kl. und kl. beh. Flecke, } \\
\text { XI I } 6 \text { entst., XI I } 8 \text { ausgetr. }\end{array}$} \\
\hline $2 \mathrm{I}$ & $22 \cdot 18$ & $-(9-12)$ & \\
\hline 22 & $\begin{array}{c}13 \\
12.8\end{array}$ & $\begin{array}{l}+9 \\
+9\end{array}$ & $\left.\begin{array}{l}\text { Beh. Fleck } \\
\text { Kl. Flecke }\end{array}\right\}$ XI I 3 entst., \\
\hline & 7 & +10 & 2 beh. Flecke) \\
\hline 3 & 4 & -14 & $\begin{array}{l}\text { Beh. Fleck, östl. kl. Fl., } \\
\text { XI } 7 \text { eingetr., XI } 20\end{array}$ \\
\hline
\end{tabular}

Rotationsperiode $309 . \mathrm{XI}_{14}$-XII Ir.

\begin{tabular}{l|l|l}
$35^{6}$ & -16 & Beh. Fleck u. östl. kl. Fl., XI 7 ein-
\end{tabular} getr., 20 ausgetr. Vgl. R. 3 I o. I 


\begin{tabular}{|c|c|c|c|}
\hline Nr. & $L$ & $b$ & Bemerkungen \\
\hline 2 & $\mid \begin{array}{c}355^{\circ}-34^{\circ} \\
349\end{array}$ & $\begin{array}{c}-\left(7^{\circ}-10^{\circ}\right) \\
-9\end{array}$ & 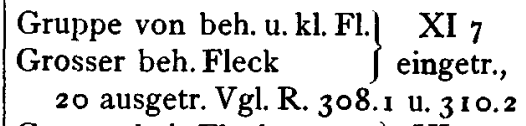 \\
\hline 3 & $\begin{array}{c}343 \\
341-331\end{array}$ & $\begin{array}{c}-7 \\
-(6-10)\end{array}$ & $\begin{array}{l}\text { Grosser beh. Fleck } \\
\text { Gruppen kl. u. beh. Flecke }\} \text { entstnd., } \\
2 \text { I ausgetr. Vgl. R. 308.I }\end{array}$ \\
\hline 4 & $315-310$ & -12 & $\begin{array}{l}\text { Gruppe m. beh. Fleck westl., XI t } 6 \\
\text { entst., } 23 \text { ausgetr. Vgl. R. } 3^{10.3}\end{array}$ \\
\hline 5 & 285 & -13 & $\begin{array}{l}\text { Beh. Fleck, XI I } 3 \text { eingtr., } 26 \text { ausgtr. } \\
\text { Vgl. R. 308.5 }\end{array}$ \\
\hline 6 & $\begin{array}{c}286-278 \\
287\end{array}$ & $\begin{array}{c}+(15.20) \\
\quad+19\end{array}$ & $\left(\begin{array}{l}\text { Stark entw. Gruppe } \\
\text { Beh.Fleck m. } 2 \text { Kern. } . \\
\text { Vgl. R. } 3 \text { I } 0.9\end{array} \quad 26\right.$ ausgetr. \\
\hline 7 & 268.263 & $-(8-14)$ & $\begin{array}{l}\text { Gruppe kl. Flecke, } \\
\text { XI } 18 \text { entst., XI } 22 \text { aufgel. }\end{array}$ \\
\hline 8 & 232 & +4 & $\begin{array}{l}\text { Beh. Fleck, östl. kl. Fl., } \\
\text { XI I } 7 \text { eingetr., XI } 24 \text { aufgel. }\end{array}$ \\
\hline 9 & 205 & $-(12-16)$ & $\begin{array}{l}\text { Gruppe kl. Flecke u. kl. beh. Fleck, } \\
\text { XI I } 9 \text { eingetr., XI } 27 \text { aufgel. }\end{array}$ \\
\hline 10 & $197-191$ & $-(15-20)$ & $\begin{array}{l}\text { Gruppe kl. Flecke, } \\
\text { XI } 2 \text { I eingetr, } 26 \text { aufgel. }\end{array}$ \\
\hline $\mathbf{I}$ & $187 \cdot 182$ & +15 & $\begin{array}{l}\text { Gruppe mit kl. beh. Fleck westl., } \\
\text { XI } 23 \text { entst., vor XI } 29 \text { aufgel. }\end{array}$ \\
\hline 12 & $\begin{array}{c}178 \\
178-170\end{array}$ & $\begin{array}{l}-I I \\
-I I\end{array}$ & $\begin{array}{l}\text { Beh. Fleck m. } 2 \text { Kern. } \\
\text { Kl. u. kl. beh. Flecke } 2 \text { r eingetr., }\end{array}$ \\
\hline & I 67 & - IO & $\begin{array}{l}\text { Beh. Fleck } \\
\text { und R. } 3 \text { I 0.1 } 5\end{array}$ \\
\hline 3 & $162-152$ & $+(8-10)$ & $\begin{array}{l}\text { Gruppe kl. und beh. Flecke, XI } 25 \\
\text { entst., XII } 5 \text { ausgetr.Vgl.R. } 3 \text { I } 0.16\end{array}$ \\
\hline 14 & I $57-154$ & - I I & $\begin{array}{l}\text { Gruppe kl. Flecke, XI } 24 \text { eingetr., } \\
26 \text { aufgel. Vgl. R. 308. I I }\end{array}$ \\
\hline 5 & 143 & $-\mathbf{I}$ & Sporad. kl. Fleck. \\
\hline & I 38 & +3 & Sporad. Pore. \\
\hline 7 & I 24 & -8 & $\begin{array}{l}\text { Ki. Fleck, wahrsch. XI } 29 \text { entst., } \\
\text { vor XII } 4 \text { aufgel. }\end{array}$ \\
\hline 18 & I 2 I & +15 & Sporad. Poren. \\
\hline 19 & 77 & - I I & $\begin{array}{l}\text { Kl. Flecke, } \\
\text { XI } 29 \text { eingetr., vor XII } 4 \text { aufgel. }\end{array}$ \\
\hline & $6 \mathrm{I}$ & -24 & Sporad. kl. Fleck. \\
\hline & 57 & +8 & $\begin{array}{l}\text { Kl. beh. Fleck, } \\
\text { XII I eingetr., vor XII } 4 \text { aufgel. }\end{array}$ \\
\hline & 33 & +10 & $\begin{array}{l}\text { Kl. Flecke, } \\
\text { XII } 5 \text { entst., nach XII } 8 \text { aufgel. }\end{array}$ \\
\hline
\end{tabular}

Zürich I 885 Aug. 12.

\begin{tabular}{c|c|c|c}
\hline Nr. & $L$ & $b$ & Bemerkungen \\
\hline 23 & $2^{\circ}$ & $+7^{\circ}$ & $\begin{array}{c}\text { Gruppe kl. Flecke, } \\
\text { XII }\end{array}$ eingetr., vor XII I 5 aufgel.
\end{tabular}

Rotationsperiode 3 ro. XII r I - I 884 I 7 .

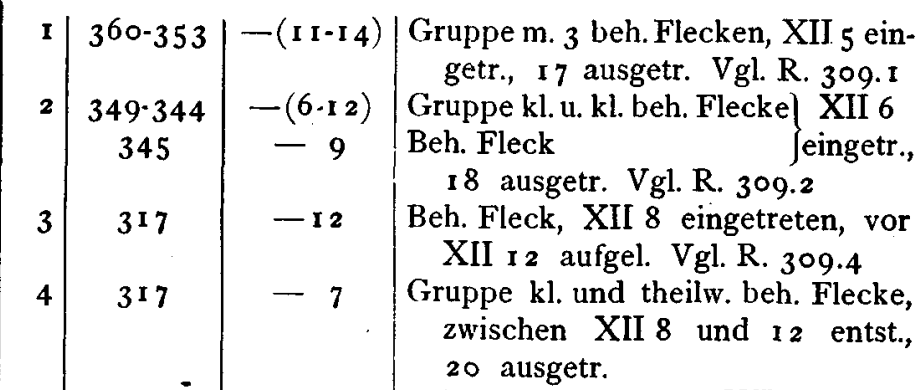

$5 \quad 3^{1} 3^{\circ}+\mathrm{r} 3$ Beb. Fleck, 2wischen XII 8 und i 2 eingetr., 20 ausgetr.

6 32 2-310 +(I 4-1 7) Gruppe mit beh. Fleck westlich, zwischen XII 8 und 12 eingetr., I 9 ausgetr.

7 301-294 +(10-16) Gruppe kl. und kl. beh. Flecke,

\begin{tabular}{l|l|l} 
& wahrsch. XII ro eingtr., 22 ausgtr.
\end{tabular}

8 284-280 -18 Gruppe kl. und kl. beh. Flecke, XII 16 entst., 22 ausgetr.

9 284-279 $+(2$ I-24) Gruppe kl. und kl. beh. Flecke, XII 12 eingetr., 23 ausgetr.

Vgl. R. 309.6 (?)

Sporad. kl. Fleck.

\begin{tabular}{l|c|c|c} 
I I & $235^{-2} 30$ & $-(5-7)$ & $\begin{array}{l}\text { Gruppe mit kl. beh. Fleck westl., } \\
\text { XII } 18 \text { entst., nach XII } 22 \text { aufgel }\end{array}$ \\
12 & $230-227$ & $+(2-4)$ & Gruppe von Poren,
\end{tabular} XII 18 entst., nach XII 22 aufgel.

$12 \mid$\begin{tabular}{l|l}
$230-227$ & $+(2-4)$ \\
& XII 20 entst., 22 aufgel.
\end{tabular}

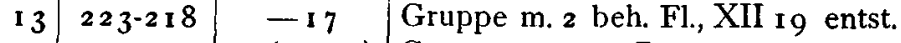

142 I0.200 $-($ I $0-17)$ Gruppe von 3 Poren,

XII 20 entst., 22 stark entw.

I 5 I 8 I-167-(6-13) Gruppe kl. und beh. Flecke, XII I 9 eingetr. Vgl. R. 309. 12

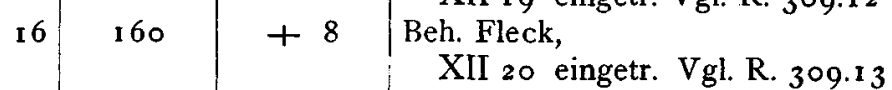

\begin{tabular}{l|l|l}
160 & 160 & Beh. Fleck, \\
& XII 20 eingetr. Vgl. R. 309.13
\end{tabular}

$\left.\begin{array}{l|c|c|l}1755 & -9 & \text { Beh. Kerngruppe } \\ 152-142 & -9 & \text { Gruppe kl. Flecke }\end{array}\right\}$ XII 20 eingetr.

Gruppe kl. Flecke
Beh. Fleck, XII 21 eingetr.

Beh. Fleck, XII 2 I eingetr.

\section{A. Wolfer.}

Beobachtungen des Planeten (251) auf der Sternwarte in Wien.

\begin{tabular}{|c|c|c|c|c|c|}
\hline 1885 & M. Z. Wien & & $\log p . \Delta$ & & $\log p .4$ \\
\hline 5 & $\begin{array}{l}12.2242 \\
103853\end{array}$ & $\begin{array}{rrr}o^{\mathrm{h}} & \mathrm{o}^{\mathrm{m}} 3^{\mathrm{s}} 8 \mathrm{8} \\
23 & 59 & 54.89\end{array}$ & $\begin{array}{l}9.120 \\
8.5^{8} \mathrm{r}_{\mathrm{n}}\end{array}$ & $\begin{array}{lrr}-7^{\circ} & 4^{\prime} & 55^{\prime \prime} \cdot 3 \\
-7 & 10 & 56.0\end{array}$ & \\
\hline
\end{tabular}

Die Position des Vergleichsterns $9^{\mathrm{m}} \cdot 5$ ( 18850$): \alpha=0^{\mathrm{h}} \mathbf{2}^{\mathrm{m}} \mathrm{I}^{\mathrm{s}} \cdot 77 \delta=-7^{\circ} 3^{\prime} 54^{\prime \prime} \cdot 7$ beruht nur auf genähertem Anschluss und bedarf einer Neubestimmung. Die Grösse des Planeten, der beim Suchen nach (217) Eudora*) gefunden wurde, ist $13^{\mathrm{m}} \cdot 5$ oder vielleicht noch etwas schwächer.

F. Palisa.

*) Eudora selbst wurde am 8. Oct. wie folgt beobachtet:

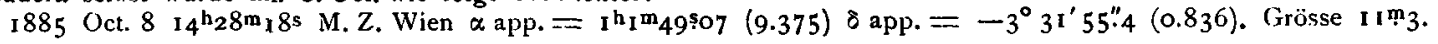

